\title{
Variable-focus Liquid Lens Based on a Laterally-integrated Thermopneumatic Actuator
}

\author{
June Kyoo Lee ${ }^{1}$, Kyung-Woo Park ${ }^{1}$, Geunbae Lim $^{3}$, Hak-Rin Kim ${ }^{1,2 \dagger}$, and Seong Ho Kong ${ }^{1,2 *}$ \\ ${ }^{1}$ Graduate School of Electrical Engineering and Computer Science, Kyungpook National University, \\ Daegu 702-701, Korea \\ ${ }^{2}$ School of Electronics Engineering, Kyungpook National University, Daegu 702-701, Korea \\ ${ }^{3}$ Department of Mechanical Engineering, Pohang University of Science and Technology, Pohang 790-784, Korea
}

(Received December 28, 2011 : revised February 3, 2012 : accepted February 14, 2012)

\begin{abstract}
We report a focal-length tunable liquid lens based on thermopneumatically driven fluidic pressure. The fluidic pressure is generated by deformation of an elastomeric diaphragm induced by thermopneumaticity from a laterally integrated microheater sealed within an air chamber. The pressure is transmitted by a confined liquid to a lens diaphragm through an internal fluid channel. The liquid filling under the lens diaphragm functions as a liquid lens for dynamic focusing with properties depending on the curvature of the deformed diaphragm. The diaphragm area of the air chamber is designed five times larger than that of the lens cavity to yield high focal-length tunability by amplified deflection of the lens diaphragm. With our method, we achieved excellent focal-length tunability from infinity (without an input current) to $4 \mathrm{~mm}$ (with an input current of $12 \mathrm{~mA}$ ) with a lens aperture diameter of $2 \mathrm{~mm}$.
\end{abstract}

Keywords: Variable-focus, Liquid lens, Thermopneumatic actuator, Elastomeric diaphragm

OCIS codes : (110.1080) Active or adaptive optics; (160.5470) Polymers; (220.3630) Lenses; (230.2090) Electro-optical devices

\section{INTRODUCTION}

Variable-focus lenses, with focal lengths that can be adjusted by manipulating the lens shape or a with a graded refractive index, have boundless possibilities for applications in a great variety of microscale optical devices such as cell phone cameras, capsule endoscopes, optical pickup heads, and microscopes [1-5]. Diverse driving forces for focal-length control have been introduced by numerous research groups. For instance, an electrowetting-based lens [6-8], dielectrically actuated lens [9, 10], fluidic-pressure-based lens [11-15], liquid-crystal lens [16-18], and polymer-gel lens [19] have been reported. Both electrowetting-based lenses and dielectrically actuated lenses use external voltage to control the shape of a refractive liquid, and they exhibit fast responses without moving mechanical parts for focal-length modulation. However, performance degradation is unavoidable with these lenses, which consist of two immiscible liquids in a lens cell. This degradation results from liquid residues on solid surfaces, condensation at low temperature, and shape hysteresis of liquid interfaces by captured charges [20-22]. Liquid crystal lenses have the complexity of electrically controlled refraction as well as the need for additional optical parts such as polarizers that avoid focusing behaviors due to optically anisotropic liquid-crystal molecules, which greatly reduce the transmitted optical power. Polymer-gel lenses are inadequate for microscale optical systems, because they require an external compressive stress for activation. The driving force of a fluidic-pressure-based tunable lens is the deformation of an elastomeric diaphragm filled with liquid. In general, refraction is controlled by either liquid injection from an external supplier or liquid flow induced with an integrated micropump. Because an external liquid supplier is impractical due to its large volume, integration of the actuator is essential for a miniaturized adaptive lens system $[14,15]$. Three miniaturized fluidic-pressure-driven lenses with integrated actuators have been previously reported [11-13]. However, the hydrogel-based fluidic-pressure lens is not convenient to use, because it is activated by chemical manipulation [11]. Moreover, an electromagnetic-actuator-based fluidic-pressure lens needs additional permanent magnets and provides limited focal-length tunability [12].

Corresponding authors: *shkong@knu.ac.kr, ${ }^{\dagger}$ rineey@ee.knu.ac.kr

Color versions of one or more of the figures in this paper are available online. 
In this study, we demonstrate a variable-focus liquid lens with an integrated actuator driven by thermopneumatically controlled fluidic pressure that affords great focal-length tunability. Specifically, the laterally integrated actuator to a lens cell converts the expansion of air heated in a sealed chamber into fluidic pressure that results in focal-length modulation without thermal crosstalk. To achieve the necessary focal-length tuning range, the structure of the proposed lens is designed according to Joule's law and Pascal's law simultaneously. The elasticity and thickness of the diaphragms at the air chamber and lens cavity, the area ratio of those diaphragms, and the optical properties of the liquid all play key roles in lens performance and so are considered with great care during the design phase. The proposed variablefocus liquid lens provides excellent focal-length tunability from infinity to $4 \mathrm{~mm}$ with a lens aperture diameter of 2 $\mathrm{mm}$. Moreover, this utilization of a thermally separated thermopneumatic actuator to drive a variable-focus liquid lens has performance superior to the earlier reported thermopneumatic liquid lens in power consumption, response time, and thermal crosstalk [13].

\section{DEVICE DESCRIPTION}

Figure 1 shows the proposed variable-focus liquid lens and its operating principle. As shown in Fig. 1(a), the lens is composed of three parts: a thermopneumatic actuator, a fluid channel, and a liquid-filled lens cavity. The thermopneumatic actuator consists of a metallic heater in a sealed air chamber with a thin elastomeric diaphragm, as shown in Fig. 1(b). When power is supplied to the heater, the elastomeric diaphragm is deflected by air pressure that is thus delivered through the fluid channel to the liquid-filled lens cavity. The corresponding diaphragm that seals the lens cavity is then deformed by the liquid pressure delivered by the incompressible fluid channel. This deformation of the lens diaphragm results in a change of focal length that is determined by the tunable deflection of the lens diaphragm,
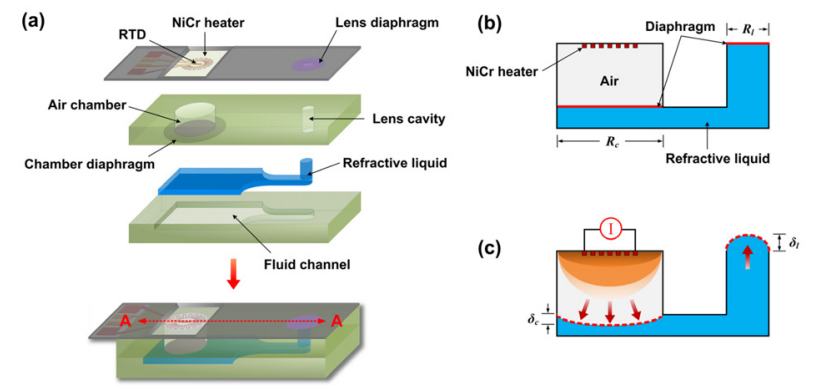

FIG. 1. (a) Proposed structures of assembled variable-focus liquid lens and its component parts. (b) Diaphragms of air chamber and liquid lens before current is applied. (c) Operating principle of variable-focus liquid lens, showing diaphragm deflections induced by thermopneumatically actuated liquid pressure after current is applied. as shown in Fig. 1(c). In order to avoid thermal crosstalk shown in the vertically integrated microlens by Zhang et al. [13], this thermopneumatic actuator and lens cell are laterally integrated using polydimethylsiloxane (PDMS) components with low thermal conductivity $(0.18 \mathrm{~W} / \mathrm{m} \cdot \mathrm{K})$ as shown in Fig. 1(a). The vertically integrated microlens showed temperature gradients of $0.2-4^{\circ} \mathrm{C}$ in the lens optical aperture. The metallic heater and the lens cavity are fabricated on a silicon wafer using micro-electro-mechanical systems (MEMS) technology, while the other parts are formed with PDMS. The fluid channel is completely filled with refractive liquid. In this study, a silicone oil (refractive index, $n_{\mathrm{oil}}=1.52$ ) is employed not only as the pressure transmitter, but also as the lens liquid. A thin insulating membrane between the rear of the metallic heater and the silicon wafer is designed to minimize the heat loss through the bulk silicon, which has a high thermal conductivity $(148 \mathrm{~W} / \mathrm{m} \cdot \mathrm{K})$. The residual thin silicon functions as a heat sink to decrease cooling speed at the air chamber as well as a mechanical support to the metallic heater.

Joule heating of the metal induces a volume change in the air chamber and in turn a pressure load on the lens, as shown in Fig. 1(c). The dependence of the pressure load, $P_{c}$, on the deflection, $\delta_{c}$, at the center of the radially symmetric chamber diaphragm is theoretically given as follows:

$$
P_{c}=E\left(\beta \Delta T-\frac{\Delta V}{V}\right)=\frac{E t}{3 R_{c}^{4}}\left(\frac{7-v}{1-v}\right) \delta_{c}^{3}
$$

where $E$ denotes the Young's modulus of the diaphragm, $\beta$ the volumetric thermal expansion coefficient, $\Delta T$ the temperature increase, $\Delta V / V$ the fractional change in volume, $t$ the diaphragm thickness, $R_{c}$ the radius of the chamber diaphragm, and $v$ the Poisson's ratio. This equation is valid for $\delta_{c} / t>5, t / R_{c}<0.15$, and diaphragms with negligible flexural rigidity [23, 24]. Because the pressure load is applied equally in all directions throughout the confined fluid, the lens diaphragm is deformed whenever the chamber diaphragm is thermopneumatically deformed, as illustrated in Fig. 1(c). To simplify the analysis, the buoyancy and gravitation of the fluid are ignored. The deflection of the lens diaphragm, $\delta_{l}$, is simply determined by Pascal's law as follows:

$$
\delta_{l}=\left(\frac{A_{c}}{A_{l}}\right) \delta_{c}=\left(\frac{R_{c}}{R_{l}}\right)^{2} \delta_{c}
$$

where $A_{c}$ and $A_{l}$ denotes the areas of the diaphragms at the air chamber and the lens cavity, respectively. $R_{l}$ is the radius of the lens diaphragm. We designed the lens with an area ratio, $A_{c} / A_{l}$, of 5.06 to induce a relatively large deformation of the lens diaphragm with a given thermopneumatic pressure. The large area ratio affords effective control over the focusing properties of the thermopneumatically actuated liquid lens. 

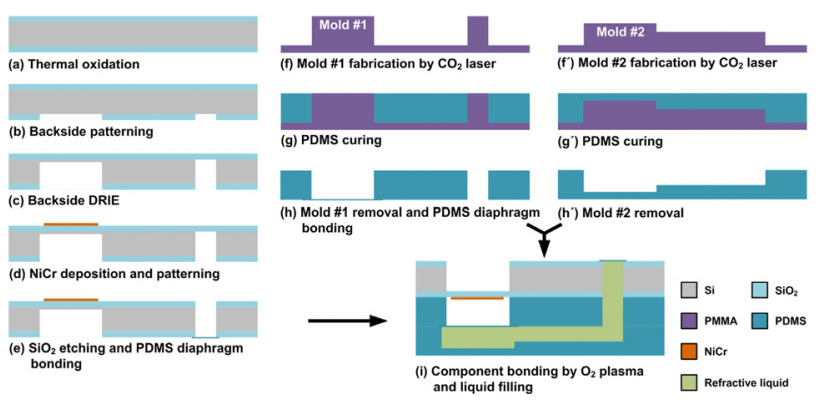

FIG. 2. Fabrication process of thermopneumatic liquid lens.

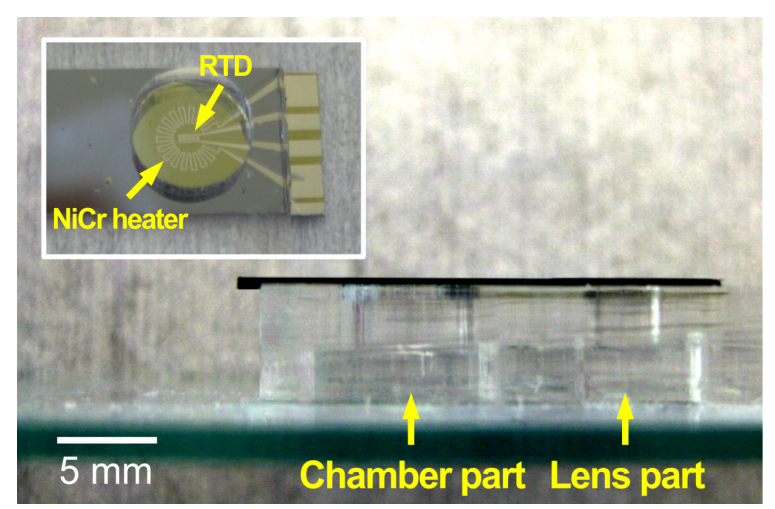

FIG. 3. Cross-sectional image of fabricated liquid lens. Enlarged overhead image of air chamber part (inset).

To demonstrate the optical performance of the variablefocus liquid lens, we fabricated the lens as illustrated schematically in Fig. 1(a). The silicon wafer, including the metallic heater and part of the lens cavity, was fabricated using thermal evaporation and deep reactive ion etching (DRIE) to form the heater and etch the cavity, respectively. The metallic heater was designed with a cogwheel shape for enhancing the Joule heating efficiency. $\mathrm{NiCr}(80 \mathrm{wt} \%$ $\mathrm{Ni}$ and $20 \mathrm{wt} \% \mathrm{Cr}$ ) was employed for the heater because of its excellent resistance to thermal corrosion. The bulk silicon on the backside of the heater was etched away using DRIE to minimize the heat loss resulting in high heating speed at the air chamber. The PDMS parts (Sylgard 184, Dow Corning, 10:1 ratio of base to curing agent) contained the chamber cavity, fluid channel, and lens cavity. These PDMS components were replicated using a poly-methyl methacrylate (PMMA) mold that was precisely machined using a carbon dioxide $\left(\mathrm{CO}_{2}\right)$ laser. Two $13-\mu \mathrm{m}$-thick elastic diaphragms for the air chamber and the lens cavity were prepared by spin coating PDMS onto a flexible polyethylene terephthalate (PET) film and subsequently were joined with the patterned PDMS structure by following a lamination process. In advance of the PDMS coating, a fluoropolymer layer (AF1600, Dupont) was formed onto the PET film to form a hydrophobic surface, which enabled the PDMS diaphragm to peel easily from the PET transfer film during the lamination process. The laminated diaphragm was firmly bonded using an oxygen $\left(\mathrm{O}_{2}\right)$ plasma treatment for
30 s. Once the silicon and PDMS parts were completed, these were aligned and then combined through successive $\mathrm{O}_{2}$ plasma bonding treatments while applying compression. Finally, the fabrication was finished by closing the fluid inlet after filling the fluid channel with silicone oil using a syringe. Figure 2 summarizes the fabrication process. Figure 3 shows images of the fabricated variable-focus liquid lens. The heater surrounds a metal rectangle that functions as a resistance temperature detector (RTD) to monitor the air chamber during lens operation.

\section{RESULTS AND DISCUSSION}

\subsection{Performance of the Thermopneumatic Actuator}

The thermopneumatic actuation of the air chamber was investigated first, because its performance is highly affected by the heat loss through the air chamber as well as by the Joule heating of the metallic heater. Measurements of the curvature were conducted using a drop-shape analyzer (DSA100, KRÜSS). The fabricated lens was positioned in a pyrostat chamber maintained at $20^{\circ} \mathrm{C}$ to exclude the influence of external temperature changes during the measurement. Figure 4(a) shows the temperature inside the air chamber and the

(a)

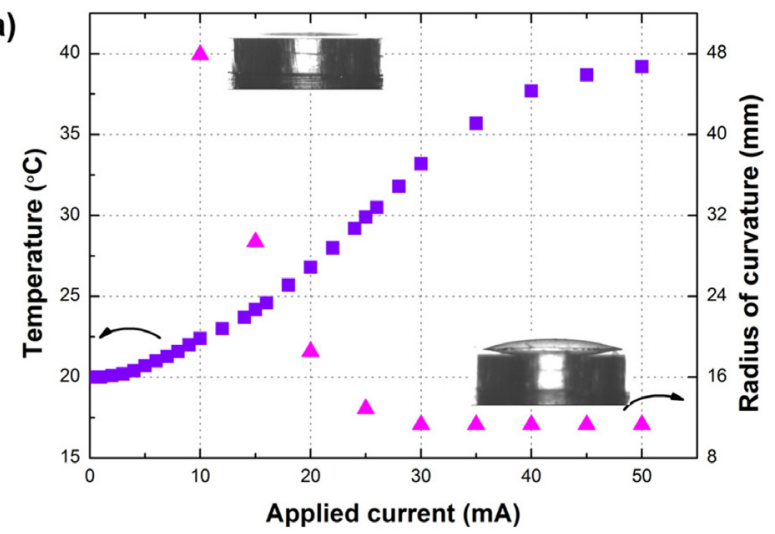

(b)

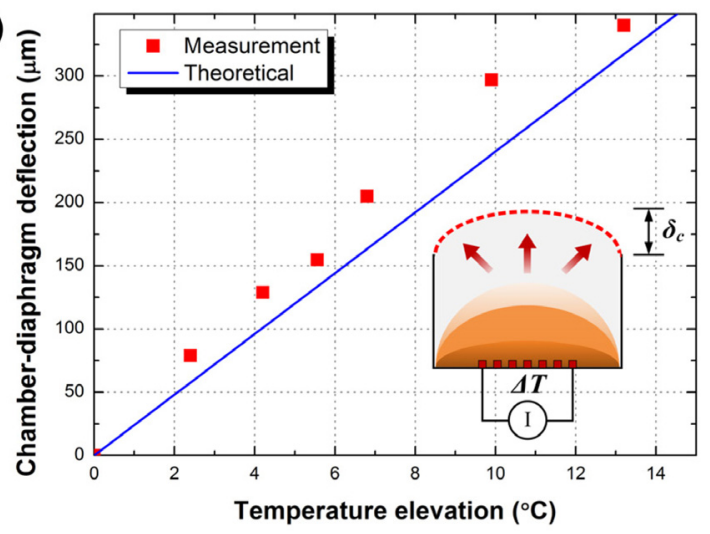

FIG. 4. (a) Chamber temperature and chamber-diaphragm curvature as functions of applied current. (b) Relationship between chamber-diaphragm deflection and chamber temperature elevation. 
curvature of the chamber diaphragm as functions of the applied current. The temperature increases parabolically, obeying Joule's law, until the current reaches $35 \mathrm{~mA}$. At this saturation point, the temperature has increased by approximately $15^{\circ} \mathrm{C}$ and the radius of curvature has reached $11.5 \mathrm{~mm}$. However, once the input current exceeds $35 \mathrm{~mA}$ the rate of temperature elevation decreases gradually due to the restriction of Joule heating by the geometry of the heater and the strong dependence of electrical conductivity on temperature in metal. The temperature saturation of the air chamber results in almost no additional deformation of the diaphragm for an input current of greater than $35 \mathrm{~mA}$. However, the maximum strain value of the PDMS diaphragm was reached at 30 $\mathrm{mA}$, before the temperature saturation of the air chamber.

Figure 4(b) shows the deformation of the chamber diaphragm as a function of the temperature elevation that was measured to check the thermal insulation of the air chamber. The diaphragm deflection induced by the temperature elevation agrees with the theoretical prediction expressed by Eq. (1) in the region where the Joule's law applies. This reveals that heat loss is minimized by the membrane structure between the silicon wafer and the metallic heater, as well as the low thermal conductivity of PDMS. Furthermore, the deflection of the air chamber diaphragm is reproducible even if accompanied by large curvature change. This reversible actuation in turn induces a consistent pressure load for deformation of the lens diaphragm. The minor discrepancy between the theoretical plot and the measured results is attributed to the differences between the actual parameters of PDMS and those assumed for numerical calculations.

Figure 5 depicts the interrelationship between the deflection of the chamber diaphragm, $\delta_{c}$, and that of the lens diaphragm, $\delta_{l}$. The measured diaphragm deflection ratio, $\delta_{l} / \delta_{c}$, is 3.18 , which means that a large change in focal-length can be obtained with a small deflection of the chamber diaphragm. However, the diaphragm deflection ratio is smaller than predicted by Eq. (2). The deviations of the measured values

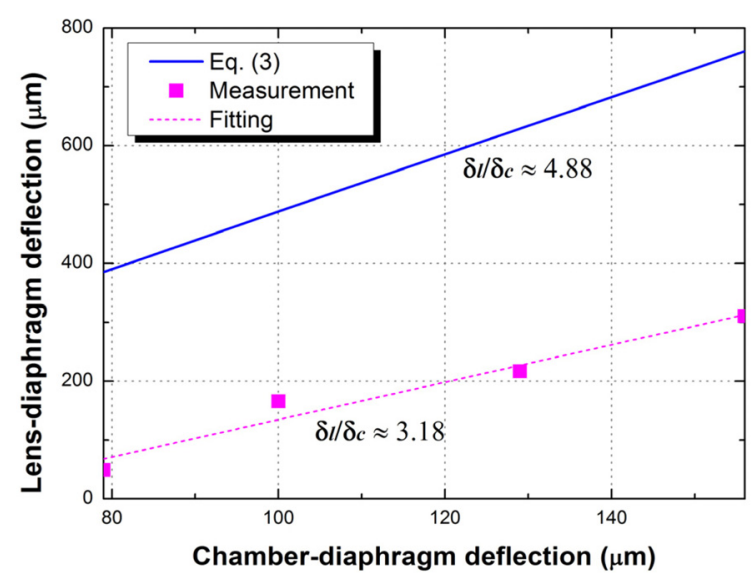

FIG. 5. The lens-diaphragm deflection versus the chamberdiaphragm deflection. from the theoretical predictions are supposed to originate with two factors: the restoring force, $F_{R}$, of the lens diaphragm; and the pressure loss throughout the fluid channel. If the diameter of the lens diaphragm is relatively small and a concentrated load is applied at the center of the lens diaphragm [25], the deflection of the diaphragm is decreased by $F_{R}$ as follows:

$$
\frac{\delta_{l}}{\delta_{c}}=\frac{A_{c} \cdot P_{c}-F_{R}}{A_{l} \cdot P_{c}-F_{R}}
$$

where

$$
F_{R}=\frac{4 \pi E t^{3}}{3\left(1-v^{2}\right) R_{l}^{2}} \cdot \delta_{l} \text {. }
$$

When the calculated $F_{R}$ value of the lens diaphragm is substituted in Eq. (3), a $\delta_{l} / \delta_{c}$ value of 4.88 is obtained. Even though the $\delta_{l} / \delta_{c}$ value is decreased by the restoring force of the lens diaphragm, the measured $\delta_{l} / \delta_{c}$ value of 3.18 is smaller yet. This means that the pressure loss induced by the restoring force of the lens diaphragm is not dominant in our system. The additional transmission loss of pressure in the fabricated system must originate with the fluid channel. Because the walls of the fluid channel are oleophilic PDMS, the silicone oil under high pressure can permeate the PDMS blocks. The resultant pressure loss can be eliminated by substituting a fluid with an extremely low PDMS affinity such as water, glycerol or immersion oil [13, 26]. However, in our experiment using water as a refractive fluid, overall focus-length tunability was decreased due to its low refractive index.

The measured rise time of the fabricated actuator was $30 \mathrm{~s}$ when the application current was $20 \mathrm{~mA}$ whereas fall time was $42 \mathrm{~s}$. Due to passive cooling of the air chamber, fall time was longer than rise time.

\subsection{Focusing Properties of the Fabricated Liquid Lens}

Figure 6(a) shows the experimental setup devised to observe the focusing performance of the fabricated thermopneumatic liquid lens. The aperture of the lens was $2 \mathrm{~mm}$ in diameter. Two objects were positioned at long and short distances, respectively, from the fabricated liquid lens mounted upon an image sensor system. Figure 6(b) shows the image observed with the unbiased condition that focused on the object positioned at the greater distance (long focal length). As shown in Fig. 6(c), with $4 \mathrm{~mA}$ of input current the lens diaphragm swelled up and then focused on the object positioned at the lesser distance (short focal length).

Figure 7 shows the radius of curvature of the lens diaphragm and the focal length determined from the curvature as functions of the applied current. As the input current increases, the focal length is reduced parabolically until it reaches 4 $\mathrm{mm}$, where the radius of curvature is minimal (about $2 \mathrm{~mm}$ ). 
(a)

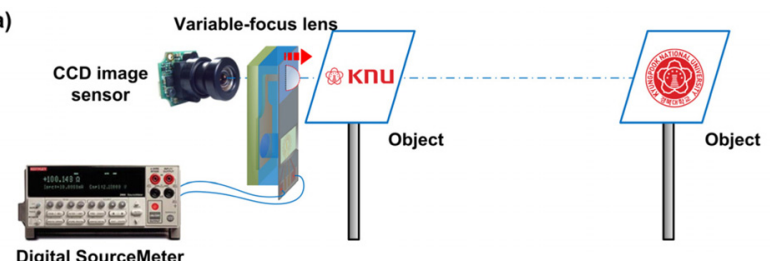

Digital SourceMeter
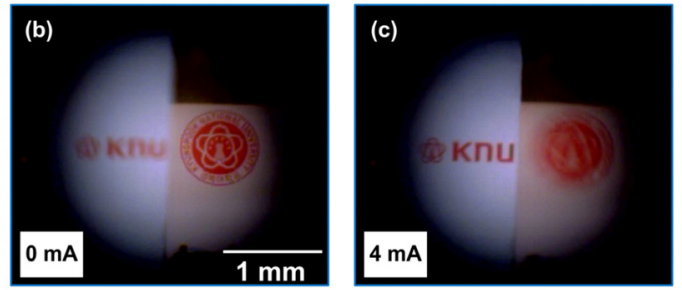

FIG. 6. (a) Experimental setup to measure focusing performances of fabricated lens. (b) CCD image obtained through liquid lens in unfocused state without input current. (c) CCD image obtained through liquid lens in focused state with input current of $4 \mathrm{~mA}$.

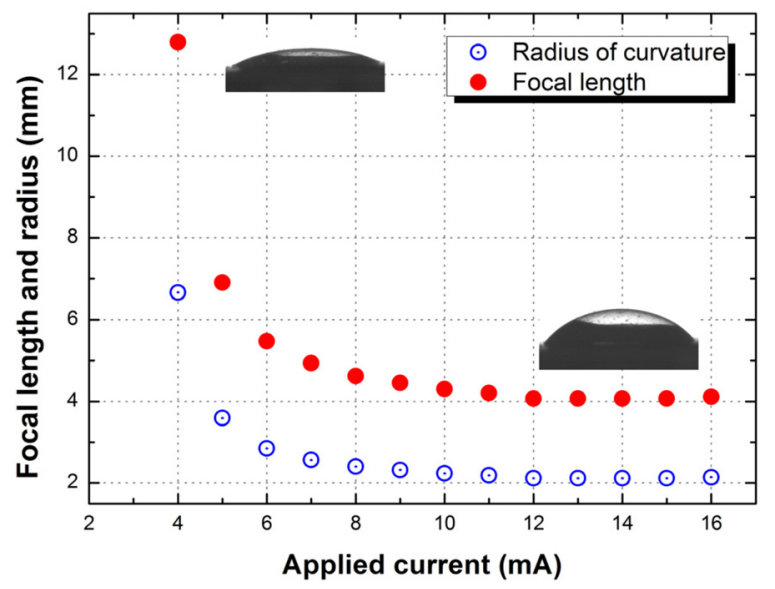

FIG. 7. Radius of curvature of lens diaphragm and resulting focal length of lens as functions of applied current.
Once the input current exceeds $12 \mathrm{~mA}$, the focal length remains almost constant. This focal-length ceiling is due to the aforementioned pressure loss through the fluid channel. The thermal influence on the focal length tunability and temperature gradient at the lens aperture were not observed in this lens. The fluid channel linking between the thermopneumatic actuator and lens cell, using low thermal conductive PDMS may separate the lens cell from a heat flux generated by the actuator. The power consumption to achieve a focal length of $4 \mathrm{~mm}$ was $144 \mathrm{~mW}$, whereas the vertically integrated thermopneumatic lens was roughly $212 \mathrm{~mW}$ [13]. The time required to obtain a focal length of $4.6 \mathrm{~mm}$ when starting from infinity with $20 \mathrm{~mA}$ of input current was 30 s. Because the response time for shortening focal length of a thermopneumatic liquid lens is dominated by the heating time of the air in the chamber, different times can be realized by optimizing the volumetric design of the air chamber or the modulation of the current density. For applications that require a quicker response, the height of the air chamber can be reduced while maintaining a large area ratio between the chamber and lens diaphragms, as expressed by Eq. (2). In addition to any change in volumetric design, the input current density can also be properly modulated with time to achieve the desired focal length. This laterally integrated thermopneumatic lens showed approximately $6 \%$ faster heating speed than that of the vertically integrated lens at the same heating power of $300 \mathrm{~mW}$ [13]. Moreover, due to heat sink through the silicon at the air chamber, the lens had much faster response than the vertically integrated lens using the glass substrate [13]. Table 1 compares the performance of variable-focus lenses according to actuation type. Most previously reported liquid lenses driven by electrical signals, such as electrowetting, dielectric forces, and piezoelectricity, have quick responses. However, these do not possess a wide range of focal-length tunability. Although fluidic-pressure-based lenses possess sufficient lens aperture size to receive much optical information, they are difficult to miniaturize because of external actuators. The prototype

TABLE 1. Performance comparison of variable-focus lenses

\begin{tabular}{|c|c|c|c|c|c|}
\hline \multicolumn{2}{|c|}{ Actuation type } & Aperture size, $\mathrm{mm}$ & Focal-length tunability, $\mathrm{mm}$ & Response time, $\mathrm{s}$ & Reference \\
\hline \multicolumn{2}{|c|}{ Electrowetting* } & 0.3 & $20-2.3$ & 0.15 & {$[27]$} \\
\hline \multicolumn{2}{|c|}{ Dielectric force* } & 3 & $34-12$ & $0.3-0.65$ & {$[10]$} \\
\hline \multicolumn{2}{|c|}{ Liquid crystal* } & 6 & $\infty-600$ & 1 & {$[28]$} \\
\hline \multirow{6}{*}{$\begin{array}{l}\text { Fluidic } \\
\text { pressure }\end{array}$} & Muscle & 20 & $172-41$ & $\mathrm{~N} / \mathrm{R}$ & {$[29]$} \\
\hline & Electromagnetic & 3 & $\mathrm{~N} / \mathrm{R}$ & $\mathrm{N} / \mathrm{R}$ & {$[12]$} \\
\hline & Piezoelectric & 3 & $\infty-79$ & 0.002 & {$[14]$} \\
\hline & Piezoelectric* & 2.5 & $500-30$ & $\mathrm{~N} / \mathrm{R}$ & {$[30]$} \\
\hline & Thermopneumatic* & 2 & $15-3$ & 32 & [13] \\
\hline & Thermopneumatic* & 2 & $\infty-4$ & 30 & This work \\
\hline
\end{tabular}

\footnotetext{
* Actuator-in-device.

N/R Not reported.
} 
of the proposed liquid lens combines novel focal-length tunability with sufficient aperture size $(2 \mathrm{~mm})$ and represents a significant advance towards a miniaturized high-performance liquid lens with an integrated actuator based on fluidic pressure. Furthermore, the thermopneumatic liquid lens is useful for an optical system that requires great focal-length tunability. A hybrid lens that incorporated the rapid and refined tunability of adaptive lenses and micro-solid lenses with the wide tunability of a thermopneumatic liquid lens might simultaneously achieve quick response, great focallength tunability, and low chromatic aberration.

\section{CONCLUSION}

We designed and fabricated a novel variable-focus liquid lens with a vertically integrated thermopneumatic actuator. The focal-length tunability of the proposed lens was determined by the area ratio of the diaphragms positioned at the actuator and lens aperture. The integrated actuator induced a deflection of the lens diaphragm that was times greater than that of the air chamber diaphragm. The novel performance of the lens was obtained by careful thermal isolation of the integrated actuator. In practice the prototype lens achieved excellent focal-length tunability from infinity to 4 $\mathrm{mm}$ with a lens diameter of $2 \mathrm{~mm}$. The proposed thermopneumatic liquid lens combined with an adaptive lens might achieve both a wide focal range and quick response.

\section{ACKNOWLEDGMENT}

This work was supported by the National Research Foundation of Korea Grant funded by the Korean Government (2011-0001078, 2011-0027306).

\section{REFERENCES}

1. B. Hendriks and S. Kuiper, "Through a lens sharply," IEEE Spectr. 41, 32-36 (2004).

2. C. Cavallotti, M. Piccigallo, E. Susilo, P. Valdastri, A. Menciassi, and P. Dario, "An integrated vision system with autofocus for wireless capsular endoscopy," Sens. Actuators A 156, $72-78$ (2009).

3. K. Y. Hung, F. G. Tseng, and T. H. Liao, "Electrostaticforce-modulated microaspherical lens for optical pickup head," J. Microelectromech. Syst. 17, 370-380 (2008).

4. S.-Y. Yim, J. H. Kim, and J. Lee, "Solid immersion lens microscope for spectroscopy of nanostructure materials," J. Opt. Soc. Korea 15, 78-81 (2011).

5. G. Kweon and Y. Choi, "Image-processing based panoramic camera employing single fisheye lens," J. Opt. Soc. Korea 14, 245-259 (2010).

6. R. Shamai, D. Andelman, B. Berge, and R. Hayes, "Water, electricity, and between... on electrowetting and its applications," Soft Matter. 4, 38-45 (2008).
7. J. K. Lee, K.-W. Park, H.-R. Kim, and S. H. Kong, "Durability enhancement of a microelectromechanical systembased liquid droplet lens," Jpn. J. Appl. Phys. 49, 06GN11 (2010).

8. L. Miccio, A. Finizio, S. Grilli, V. Vespini, M. Paturzo, S. D. Nicola, and P. Ferraro, "Tunable liquid microlens arrays in electrode-less configuration and their accurate characterization by interference microscopy," Opt. Express 17, 2487-2499 (2009).

9. H. Ren, S. Xu, and S. T. Wu, "Deformable liquid droplets for optical beam control," Opt. Express 18, 11904-11910 (2010).

10. C. C. Cheng and J. A. Yeh, "Dielectrically actuated liquid lens," Opt. Express 15, 7140-7145 (2007).

11. L. Dong, A. K. Agarwal, D. J. Beebe, and H. R. Jiang, "Adaptive liquid microlenses activated by stimuli-responsive hydrogels," Nature 442, 551-554 (2006).

12. S. W. Lee and S. S. Lee, "Focal tunable liquid lens integrated with an electromagnetic actuator," Appl. Phys. Lett. 90, 121129 (2007).

13. W. Zhang, K. Aljasem, H. Zappe, and A. Seifert, "Completely integrated, thermo-pneumatically tunable microlens," Opt. Express 19, 2347-2362 (2011).

14. H. Oku and M. Ishikawa, "High-speed liquid lens with $2 \mathrm{~ms}$ response and $80.3 \mathrm{~nm}$ root-mean-square wavefront error," Appl. Phys. Lett. 94, 221108 (2009).

15. P. M. Moran, S. Dharmatilleke, A. H. Khaw, K. W. Tan, M. L. Chan, and I. Rodriguez, "Fluidic lenses with variable focal length," Appl. Phys. Lett. 88, 041120 (2006).

16. M. Ye, B. Wang, T. Takahashi, and S. Sato, "Properties of variable-focus liquid crystal lens and its application in focusing system," Opt. Rev. 14, 173-175 (2007).

17. M. Hain, R. Glöckner, S. Bhattacharya, D. Dias, S. Stankovic, and T. Tschudi, "Fast switching liquid crystal lenses for a dual focus digital versatile disk pickup," Opt. Comm. 188, 291-299 (2001).

18. A. F. Naumov, G. D. Love, M. Y. Loktev, and F. L. Vladimirov, "Control optimization of spherical modal liquid crystal lenses," Opt. Express 4, 344-352 (1999).

19. G. Beadie, M. L. Sandrock, M. J. Wiggins, R. S. Lepkowicz, J. S. Shirk, M. Ponting, Y. Yang, T. Kazmierczak, A. Hiltner, and E. Baer, "Tunable polymer lens," Opt. Express 16, 1184711857 (2008).

20. J. M. Oh, S. H. Ko, and K. H. Kang, "Analysis of electrowetting-driven spreading of a drop in air," Phys. Fluids 22, 032002 (2010).

21. M. Maillard, J. Legrand, and B. Berge, "Two liquids wetting and low hysteresis electrowetting on dielectric applications," Langmuir 25, 6162-6167 (2009).

22. C. C. Cheng, C. A. Chang, and J. A. Yeh, "Variable focus dielectric liquid droplet lens," Opt. Express 14, 4101-4106 (2006).

23. F. Amirouche, Y. Zhou, and T. Johnson, "Current micropump technologies and their biomedical applications," Microsyst. Technol. 15, 647-666 (2009).

24. M. D. Giovanni, Flat and Corrugated Diaphragm Design Handbook (Marcel Dekker, New York, USA, 1982).

25. A. C. Ugural, Stresses in Plates and Shells (WCB McGrawHill, Boston, USA, 1999).

26. J. N. Lee, C. Park, and G. M. Whitesides, "Solvent compatibility of poly(dimethylsiloxane)-based microfluidic devices," 
Anal. Chem. 75, 6544-6554 (2003).

27. F. Krogmann, W. Mönch, and H. Zappe, "Electrowetting for tunable microoptics," J. Microelectromech. Syst. 17, 15011512 (2008).

28. H. Ren, Y. H. Fan, S. Gauza, and S. T. Wu, "Tunablefocus flat liquid crystal spherical lens," Appl. Phys. Lett. 84, 4789-4791 (2004).
29. D. Y. Zhang, V. Lien, Y. Berdichevsky, J. Choi, and Y. H. Lo, "Fluidic adaptive lens with high focal length tunability," Appl. Phys. Lett. 82, 3171-3172 (2003).

30. F. Schneider, J. Draheim, C. Müller, and U. Wallrabe, "Optimization of an adaptive PDMS-membrane lens with an integrated actuator," Sens. Actuators A 154, 316-321 (2009). 